\title{
Universal correlations of one-dimensional electrons at low density
}

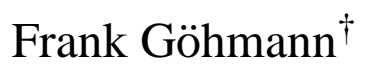 \\ Theoretische Physik I, Universität Bayreuth, 95440 Bayreuth, \\ Germany
}

\begin{abstract}
We summarize results on the asymptotics of the two-particle Green functions of interacting electrons in one dimension. Below a critical value of the chemical potential the Fermi surface vanishes, and the system can no longer be described as a Luttinger liquid. Instead, the non-relativistic Fermi gas with infinite point-like repulsion becomes the universal model for the long-wavelength, low temperature physics of the one-dimensional electrons. This model, which we call the impenetrable electron gas, allows for a rigorous mathematical treatment. In particular, a so-called determinant representation for the two particle Green function could be derived. This determinant representation is related to an integrable classical evolution equation and to a Riemann-Hilbert problem, that enable the exact calculation of the asymptotics of the two-particle Green functions.
\end{abstract}

PACS: 05.30.Fk; 71.10.Pm; 71.27.+a

\footnotetext{
${ }^{\dagger}$ e-mail: Frank.Goehmann@uni-bayreuth.de
} 


\section{Correlation functions}

Correlation functions are (thermal) expectation values of products of field operators. As a typical example we may consider the two-particle Green function $G_{\alpha \beta}(x, t)=\left\langle\Psi_{\alpha}(x, t) \Psi_{\beta}^{+}(0,0)\right\rangle$. Here $\Psi_{\alpha}$ and $\Psi_{\alpha}^{+}$are annihilation and creation operators of electrons with spin $\alpha=\uparrow, \downarrow$. The brackets denote the grand canonical ensemble average. The two-particle Green function $G_{\alpha \beta}(x, t)$ gives the probability to find an electron at $(x, t)$ provided there was an electron at $(0,0)$. It describes the propagation of an electron in a medium of other electrons, which, in general, is characterized by a temperature $T$, a chemical potential $\mu$ and a magnetic field $B$.

Within the frame work of the linear response theory correlation functions provide the link between the microscopic and the macroscopic properties of matter. Correlation functions are measurable in experiments. The two-particle Green function, for instance, measures the optical absorption.

Only a few mathematically exact results on correlation functions of interacting systems are known. In fact, the example discussed in this contribution is the first example of a direct Bethe ansatz calculation of the asymptotics of correlations of interacting electrons. The calculation was performed for the so-called impenetrable electron gas model, which is the infinite repulsive coupling limit of Yang's model of electrons with point-like pair interaction [1, 2]. As we shall argue below, our results are, in spite of being obtained for a specific model, applicable to a whole class of models of interacting electrons in one dimension, and are, in this sense, universal.

In the first part of this text we shall explain our ideas about the universality of the long-wavelength, low-temperature asymptotics of electronic correlations in the gas phase [3]. We shall start with the paradigmatic Hubbard model, and then argue that certain modifications of the interaction do not change the longwavelength, low-temperature physics of the model. In the appropriate scaling limit all modified Hamiltonians lead to the same effective model. The second part of this text is devoted to a summary of our calculation of the asymptotics of the two-particle Green functions of the impenetrable electron gas model [4, 5].

\section{The Hubbard model in the gas phase}

The density $D$ of non-interacting, one-dimensional spin- $\frac{1}{2}$ Fermions on a lattice is given by the integral over the Fermi weight,

$$
D=\frac{2}{\pi} \int_{0}^{\pi} d p \frac{1}{e^{(\varepsilon(p)-\mu) / T}+1} .
$$

Here $\varepsilon(p)$ is the dispersion of the Fermions, $T$ denotes the temperature and $\mu$ the chemical potential. Let us assume $\varepsilon(p)$ to be monotonically increasing and 
bounded from below. If the chemical potential is smaller than a critical value, $\mu<\mu_{c}=\min _{p>0} \varepsilon(p)$, then $D$ vanishes in the zero temperature limit $T \rightarrow 0+$. For $\mu>\mu_{c}$, on the other hand, the density $D$ approaches a finite positive value as $T \rightarrow 0+$. This means that the system undergoes a phase transition at $T=0$ as a function of the chemical potential. The critical point is at $\mu=\mu_{c}$. Assuming that $\varepsilon(p)=\mu_{c}+p^{2}+O\left(p^{4}\right)$, we obtain

$$
D=\frac{2}{\pi} \sqrt{\mu-\mu_{c}}
$$

for $\mu_{c}<\mu<\mu_{c}+\delta, \delta \ll 1$.

As we shall see below by considering a representative example this scenario remains unchanged if the Fermions interact. For interacting one-dimensional Fermions the phase with $\mu>\mu_{c}$, for which the density at $T=0$ is finite, is called the Luttinger liquid phase [6, [, 8]. This phase is quite familiar to many physicists. A Luttinger liquid may be understood as a one-dimensional metal. The correlations in the Luttinger liquid are dominated by fluctuations around the Fermi surface. Their power law decay at zero temperature is described by conformal field theory [9]. For small finite temperature conformal field theory predicts exponentially decaying correlations. One has to employ a conformal mapping from the complex plane to a strip of finite width. As a result the rate of exponential decay is defined by conformal dimensions.

The phase with $\mu<\mu_{c}$ so far attracted less attention. This phase is trivial at $T=0$, since the density $D$ vanishes for $T=0$. The density becomes positive for positive temperature, and is typically exponentially small as long as the temperature remains small. The ideal gas law holds. This suggested the name 'gas phase' to us. The gas phase may be interpreted as a one-dimensional semi conductor or insulator. Correlations in the gas phase behave essentially different compared to those in the Luttinger liquid phase. This is the subject of this text.

In order to get a better understanding of the gas phase of interacting systems let us consider the Hubbard model as an example,

$$
H_{H}=-\sum_{j=1}^{L}\left(c_{j+1, \sigma}^{+} c_{j, \sigma}+c_{j, \sigma}^{+} c_{j+1, \sigma}\right)+U \sum_{j=1}^{L} n_{j \uparrow} n_{j \downarrow}-\mu \sum_{j=1}^{L}\left(n_{j \uparrow}+n_{j \downarrow}\right) .
$$

Here the canonical Fermi operators $c_{j, \sigma}^{+}, c_{j, \sigma}$ are creation and annihilation operators of electrons at site $j$ of a one-dimensional, periodically closed chain of $L$ lattice sites, and $n_{j, \uparrow}$ and $n_{j, \downarrow}$ are the corresponding particle number operators. $U$ is the strength of the repulsive interaction.

The eigenvalue problem of the Hubbard Hamiltonian can be solved [10] by means of the nested Bethe ansatz. This allows us to test our ideas about the gas 
phase quantitatively. The energy levels for the $N$ electron system are

$$
E=2 \sum_{j=1}^{N}\left(1-\cos k_{j}\right)-(\mu+2) N
$$

where the charge momenta $k_{j}$ are solutions of the Lieb-Wu equations [10]. Clearly the first term on the right hand side of (4) is non-negative. Hence, if $\mu<\mu_{c}=-2$, the energies of all eigenstates become non-negative, and the absolute ground state is the empty lattice. For $\mu>-2$, on the other hand, the energy can be lowered by filling states with small $k$ 's. Since $k_{j+1}-k_{j} \sim 1 / L$, this leads to a finite density of electrons in the ground state as $L \rightarrow \infty$. We conclude that the Hubbard model is in the gas phase for $\mu<-2$ and in the Luttinger liquid phase else. Note that the asymptotics of correlation functions of the Hubbard model in the Luttinger liquid phase was obtained in [11, 12].

Another way of understanding the transition from the gas phase to the Luttinger liquid phase is by looking at the integral equations that describe the Hubbard model in the thermodynamic limit (see e.g. [13]). At zero temperature and zero magnetic field the dressed energy $\kappa(k)$ of the elementary charge excitations is determined by the integral equation

$$
\kappa(k)=-2 \cos (k)-\mu+\int_{-Q}^{Q} d k^{\prime} \cos \left(k^{\prime}\right) R\left(\sin \left(k^{\prime}\right)-\sin (k)\right) \kappa\left(k^{\prime}\right),
$$

where the limits of integration depend on the chemical potential through the condition

$$
\kappa(Q)=0
$$

The integral kernel $R$ is given by

$$
R(x)=\int_{-\infty}^{\infty} \frac{d \omega}{2 \pi} \frac{e^{i \omega x}}{1+e^{U|\omega| / 2}} .
$$

Similarly, the density $\rho(k)$ of elementary charge excitations is obtained from the integral equation

$$
\rho(k)=\frac{1}{2 \pi}+\int_{-Q}^{Q} d k^{\prime} \cos (k) R\left(\sin \left(k^{\prime}\right)-\sin (k)\right) \rho\left(k^{\prime}\right) .
$$

$\rho(k)$ determines the density of the electrons at zero temperature,

$$
D=\int_{-Q}^{Q} d k \rho(k)
$$


Since $\rho(k)$ is positive, $D$ is equal to zero if and only if $Q=0$. Hence, it follows from (5) and (6) that $\mu_{c}=-2$. Moreover, the equations (5)-(9) are easily solved for small Q. From the solution we obtain

$$
D=\frac{1}{\pi} \sqrt{\mu-\mu_{c}}
$$

for $\mu_{c}<\mu<\mu_{c}+\delta, \delta \ll 1$.

Thus the qualitative picture is the same for free and for interacting electrons. Comparing (2) and (10), however, we see that the results for the electron density close to the critical point $\mu=\mu_{c}$ differ by a factor of two. This difference may be interpreted as a signature of a phenomenon called spin-charge separation (see, for instance, [8]): The elementary charge excitations of the Hubbard model at finite positive $U$ are spinless, hence the density is smaller by a factor of two.

To complete our understanding of the gas phase of the one-dimensional Hubbard model let us consider the low temperature thermodynamics of the gas phase. The thermodynamics of the Hubbard model was first considered by Takahashi [14]. The limiting case we are interested in, however, was not studied in Takahashi's paper. Takahashi expressed the Gibbs free energy $\omega=-P(P$ pressure $)$ in terms of the dressed energies $\kappa(k), \varepsilon_{n}(\Lambda), \varepsilon_{n}^{\prime}(\Lambda)$, of elementary excitations at finite temperature. $\kappa(k)$ is the dressed energy of particle (or hole) excitations, $\varepsilon_{n}(\Lambda)$ describes spin excitations and $\varepsilon_{n}^{\prime}(\Lambda)$ so-called $k-\Lambda$ strings [13]. All $k-\Lambda$ strings are gapped [14]. They do not contribute to the low-temperature thermodynamic properties of the Hubbard model [15] and drop out of the equation for the pressure, which simplifies to

$$
P=\frac{T}{2 \pi} \int_{-\pi}^{\pi} d k \ln \left(1+e^{-\frac{k(k)}{T}}\right) .
$$

Similarly, the integral equations for the dressed energies at low temperature become

$$
\begin{aligned}
\kappa(k)= & -\mu-2 \cos k-T \sum_{n=1}^{\infty}\left([n] \ln \left(1+e^{-\frac{\varepsilon_{n}}{T}}\right)\right)(\sin k), \\
\ln \left(1+e^{\frac{\varepsilon_{n}(\Lambda)}{T}}\right)= & -\int_{-\pi}^{\pi} d k \cos k a_{n}(\Lambda-\sin k) \ln \left(1+e^{-\frac{\kappa(k)}{T}}\right) \\
& +\sum_{m=1}^{\infty}\left(A_{n m} \ln \left(1+e^{-\frac{\varepsilon_{n}}{T}}\right)\right)(\Lambda),
\end{aligned}
$$

where $n=1,2,3, \ldots$ in equation (13), and

$$
a_{n}(\Lambda)=\frac{n U / 4 \pi}{(n U / 4)^{2}+\Lambda^{2}} .
$$


$[n]$ and $A_{n m}$ are integral operators defined by

$$
\begin{aligned}
([0] f)(\Lambda) & =f(\Lambda), \\
([n] f)(\Lambda) & =\int_{-\infty}^{\infty} d \Lambda^{\prime} a_{n}\left(\Lambda-\Lambda^{\prime}\right) f\left(\Lambda^{\prime}\right), n=1,2, \ldots \\
A_{n m} & =\sum_{j=1}^{\min \{n, m\}}([|n-m|+2(j-1)]+[|n-m|+2 j]) .
\end{aligned}
$$

The gas phase is characterized by the absence of a Fermi surface for $\kappa(k)$. Thus $\kappa(k)$ is positive in the zero temperature limit, and the first term on the right hand side of (13) becomes exponentially small in $T$. Dropping this term, the equations (13) decouple from (12). Since the equations become independent of $\Lambda$, it is not hard to solve them. The solution, $\exp \left\{\varepsilon_{n}(\Lambda) / T\right\}=n(n+2)$, is the same as in the infinite coupling limit $U \rightarrow \infty$ (cf. e.g. [16]). Inserting this solution into (12) we obtain

$$
\kappa(k)=-\mu-2 \cos k-T \ln 2 .
$$

Our initial assumption, that $\lim _{T \rightarrow 0} \kappa(k)>0$ holds for all $k$, is self-consistent, if $\mu+2<0$, which is precisely the condition for being in the gas phase stated above. With (18) the low temperature expression for the pressure becomes

$$
P=\frac{T}{2 \pi} \int_{-\pi}^{\pi} d k \ln \left(1+2 e^{\frac{\mu+2 \cos k}{T}}\right) \approx \sqrt{\frac{T}{\pi}} e^{\frac{\mu+2}{T}},
$$

and we see that the density $D=\partial P / \partial \mu$ and the pressure $P$ are related by the ideal gas law,

$$
P=T D .
$$

There are two important lessons to learn from our simple calculation. First, the low temperature limit in the gas phase works the same way as the strong coupling limit at finite temperatures. Second, the low temperature Gibbs free energy $\omega=$ $-P$ in the gas phase shows no signature of the discreteness of the lattice. It is the same as for the impenetrable electron gas (see below), which is a continuum model. This agrees well with our intuitive understanding of the gas phase at low temperature: (i) The mean free path $(=1 / D)$ of the electrons is large compared to the lattice spacing (which we set equal to unity so far). (ii) Their kinetic energy is of the order $T$. Hence, the effective repulsion is large for $T \ll U$. (iii) The ideal gas law holds at low temperature. 


\section{Scaling}

The above arguments show that only electrons with small momenta, corresponding to long wavelengths contribute to the low-temperature properties of the Hubbard model in the gas phase. Thus the Hubbard model in the gas phase at low temperature is effectively described by its continuum limit. In order to perform the continuum limit we have to introduce the lattice spacing $\Delta$ and coordinates $x=\Delta n$ connected with the $n$th lattice site. The total length of the system is $\ell=\Delta L$. The continuum limit is the limit $\Delta \rightarrow 0$ for fixed $\ell$. In this limit we obtain canonical field operators $\Psi_{\sigma}(x)$ for electrons of spin $\sigma$ as

$$
\Psi_{\sigma}(x)=\lim _{\Delta \rightarrow 0} c_{n, \sigma} / \sqrt{\Delta} .
$$

Let us perform the rescaling

$$
T_{H}=\Delta^{2} T, \mu_{H}+2=\Delta^{2} \mu, k_{H}=\Delta k, t_{H}=t / \Delta^{2}, B_{H}=\Delta^{2} B,
$$

where $k$ denotes the momentum, $t$ the time and $B$ the magnetic field, which we shall incorporate below. The index ' $H$ ' refers to the Hubbard model. Then, in the limit $\Delta \rightarrow 0$, we find

$$
H_{H} / T_{H}=H / T .
$$

Here $H$ is the Hamiltonian for continuous electrons with delta interaction,

$$
\begin{array}{r}
H=\int_{-\ell / 2}^{\ell / 2} d x\left\{\left(\partial_{x} \Psi_{\alpha}^{+}(x)\right) \partial_{x} \Psi_{\alpha}(x)+\frac{U}{\Delta} \Psi_{\uparrow}^{+}(x) \Psi_{\downarrow}^{+}(x) \Psi_{\downarrow}(x) \Psi_{\uparrow}(x)\right. \\
\left.-\mu \Psi_{\alpha}^{+}(x) \Psi_{\alpha}(x)\right\} .
\end{array}
$$

Note that the coupling $c_{1}=U / \Delta$ of the continuum model goes to infinity! This is a peculiarity of the one-dimensional system. The effective interaction in the low density phase becomes large. Similar scaling arguments lead to an effective coupling $c_{2}=U$ in two dimensions and to $c_{3}=\Delta U$ in three dimensions, i.e. unlike one-dimensional electrons three-dimensional electrons in the gas phase are free.

\section{Universality}

What happens to more general Hamiltonians in the continuum limit? Let us consider Hamiltonians of the form $H_{G}=H_{H}+V$, where $H_{H}$ is the Hubbard Hamiltonian and $V$ contains additional short range interactions. We shall assume that $V$ is a sum of local terms $V_{j}$ which preserve the particle number. Then $V_{j}$ contains as 
many creation as annihilation operators, and the number of field operators in $V_{j}$ is even. We shall further assume that $V_{j}$ is hermitian and space parity invariant.

According to equation (21) every field $c_{j, \sigma}$ on the lattice contributes a factor of $\Delta^{1 / 2}$ in the continuum limit. One factor of $\Delta$ is absorbed by the volume element $d x=\Delta$, when turning from summation to integration. Thus, if $V_{j}$ contains 8 or more field operators, then $V \sim \Delta^{3}$ and $V / T_{H}$ vanishes. If $V_{j}$ contains 6 field operators, then at least two of the creation operators and two of the annihilation operators must belong to different lattice sites, since otherwise $V_{j}=0$. A typical term is, for instance, $V_{j}=c_{j, \uparrow}^{+} c_{j, \downarrow}^{+} c_{j+1, \uparrow}^{+} c_{j+1, \uparrow} c_{j, \downarrow} c_{j, \uparrow}$. In the continuum limit we have $c_{j+1, \uparrow}=\Delta^{1 / 2} \Psi_{\uparrow}(x)+\Delta^{3 / 2} \partial_{x} \Psi_{\uparrow}(x)+O\left(\Delta^{5 / 2}\right)$. Hence, the leading term vanishes due to the Pauli principle. The next to leading term acquires an additional power of $\Delta$. We conclude that $V \sim \Delta^{3}$ and thus $V / T_{H} \rightarrow 0$.

If $V_{j}$ contains 4 fields, then

$$
V \sim \Delta^{2} \Psi_{\uparrow}^{+}(x) \Psi_{\downarrow}^{+}(x) \Psi_{\downarrow}(x) \Psi_{\uparrow}(x)+O\left(\Delta^{4}\right) .
$$

Here the first term on the right hand side is the density-density interaction of the electron gas. In order to arrive at the impenetrable electron gas model the coefficient in front of this term has to be positive. Note that there are no terms of the order of $\Delta^{3}$ on the right hand side of (25) and thus no other terms than the first one in the continuum limit. Terms of the order of $\Delta^{3}$ would contain precisely one spatial derivative. They are ruled out, since they would break space parity.

Considering the case, when $V_{j}$ contains 2 fields, we find, except for the kinetic energy and the chemical potential term, terms which correspond to a coupling to an external magnetic field $B_{H}$. For these terms to be finite in the continuum limit we have to rescale the magnetic field as $B_{H}=\Delta^{2} B$ (cf. equation (22)).

Our considerations show that the impenetrable electron gas model with magnetic field,

$$
H_{B}=H+B \int_{-\ell / 2}^{\ell / 2} d x \Psi_{\alpha}^{+}(x) \sigma_{\alpha \beta}^{z} \Psi_{\beta}(x)
$$

is indeed the universal model (for small $T$ ) for the gas phase of one-dimensional lattice electrons with repulsive short-range interaction.

\section{Impenetrable electrons}

The impenetrable electron gas is the infinite coupling limit of the electron gas with repulsive delta interaction $(\Delta \rightarrow 0$ in (26)), which was the first model solved by nested Bethe ansatz [1, 2]. The pressure of the system as a function of $T, \mu$ and $B$ 
is known explicitly [16],

$$
P=\frac{T}{2 \pi} \int_{-\infty}^{\infty} d k \ln \left(1+e^{\frac{\mu+B-k^{2}}{T}}+e^{\frac{\mu-B-k^{2}}{T}}\right),
$$

and may serve as thermodynamic potential. The expression (27) is formally the same as for a gas of free spinless Fermions with effective (temperature dependent) chemical potential $\mu_{e f f}=\mu+T \ln (2 \cosh B / T)$. Hence the Fermi surface vanishes for $\lim _{T \rightarrow 0} \mu_{\text {eff }}=\mu+|B|<0$. The finite temperature correlation functions of the impenetrable electron gas depend crucially on the sign of $\mu_{e f f}$. This allows us to define the gas phase at finite temperature by the condition $\mu_{e f f}<0$, which is also sufficient for deriving the ideal gas law (20) from the low temperature limit of (27). Note that for zero magnetic field and small temperature equation (27) coincides with the right hand side of (19).

The time and temperature dependent (two-point) Green functions are defined as

$$
\begin{aligned}
& G_{\uparrow \uparrow}^{+}(x, t)=\frac{\operatorname{tr}\left(e^{-H_{B} / T} \Psi_{\uparrow}(x, t) \Psi_{\uparrow}^{+}(0,0)\right)}{\operatorname{tr}\left(e^{-H_{B} / T}\right)}, \\
& G_{\uparrow \uparrow}^{-}(x, t)=\frac{\operatorname{tr}\left(e^{-H_{B} / T} \Psi_{\uparrow}^{+}(x, t) \Psi_{\uparrow}(0,0)\right)}{\operatorname{tr}\left(e^{-H_{B} / T}\right)} .
\end{aligned}
$$

For the impenetrable electron gas these correlation functions were represented as determinants of Fredholm integral operators in [17, 18]. The determinant representation provides a powerful tool to study their properties analytically.

In [4, 5] the determinant representation was used to derive a nonlinear partial differential equation for two classical auxiliary fields, which determine the correlation functions. This partial differential equation is closely related to the Heisenberg equation of the quantum Hamiltonian (24). It is called the separated nonlinear Schrödinger equation. Together with a corresponding Riemann-Hilbert problem it determines the large-time, long-distance asymptotics of the correlators (28), (29) (for details see the following sections). In [4, 5] the asymptotics $x, t \rightarrow \infty$ was calculated for fixed ratio $k_{0}=x / 2 t$. The crucial parameter for the asymptotics is the average number of particles $x D$ in the interval $[0, x]$. If $x$ is large but $x D \ll 1$ (i.e. $T$ small), an electron propagates freely from 0 to $x$, and the correlation functions (28), (29) are those of free Fermions,

$$
\begin{aligned}
& G_{f}^{+}(x, t)=\frac{e^{-\frac{\mathrm{i} \pi}{4}}}{2 \sqrt{\pi}} t^{-\frac{1}{2}} e^{\mathrm{i} t(\mu-B)} e^{\frac{\mathrm{i} x^{2}}{4 t}} \\
& G_{f}^{-}(x, t)=\frac{e^{\frac{\mathrm{i} \pi}{4}}}{2 \sqrt{\pi}} e^{\frac{\left(\mu-B-k_{0}^{2}\right)}{T}} t^{-\frac{1}{2}} e^{-\mathrm{i} t(\mu-B)} e^{-\frac{\mathrm{i} x^{2}}{4 t}}
\end{aligned}
$$


The true asymptotic region is characterized by a large number $x D$ of particles in the interval $[0, x]$, specifically, $x D \gg z_{c}^{-1}$, where $z_{c}=\left(T^{3 / 4} e^{-k_{0}^{2} / 2 T}\right) /\left(2 \pi^{1 / 4} k_{0}^{3 / 2}\right)$. If the latter condition is satisfied, the correlation functions decay due to multiple scattering. The cases $B>0$ and $B \leq 0$ have to be treated separately. For $B>0$ a critical line, $x=4 t \sqrt{B}$, separates the $x$ - $t$ plane into a time and a space like regime. The asymptotics (for small $T$ ) in these respective regimes are:

Time like regime $(x<4 t \sqrt{B})$ :

$$
G_{\uparrow \uparrow}^{ \pm}(x, t)=G_{f}^{ \pm}(x, t) \frac{t^{\mp \mathrm{i} v\left(z_{c}\right)} e^{-x D_{\downarrow}}}{\sqrt{4 \pi z_{c} x D_{\downarrow}}},
$$

where

$$
v\left(z_{c}\right)=-\frac{2 D_{\downarrow} k_{0}^{3 / 2} e^{-k_{0}^{2} / 2 T}}{\pi^{1 / 4} T^{5 / 4}} \quad, \quad D_{\downarrow}=\frac{1}{2} \sqrt{\frac{T}{\pi}} e^{(\mu+B) / T} .
$$

$D_{\downarrow}=\partial P / \partial(\mu+B)$ is the low temperature expression for the density of down-spin electrons.

Space like regime $(x>4 t \sqrt{B})$ :

$$
G_{\uparrow \uparrow}^{ \pm}(x, t)=G_{f}^{ \pm}(x, t) t^{\mp \mathrm{iv}\left(\gamma^{-1}\right)} e^{-x D_{\downarrow}},
$$

where

$$
v\left(\gamma^{-1}\right)=-\frac{e^{\left(3 B+\mu-k_{0}^{2}\right) / T}}{2 \pi} .
$$

For $B \leq 0$ there is no distinction between time and space like regimes. The asymptotics is given by (34).

It is fair to mention here that the calculation of the asymptotics (32), (34) is rather lengthy. Equations (32) and (34) are asymptotic expansions in $t$ consisting of an exponential factor, a power law factor and a constant factor. Note that the method employed in [5] allows for a systematic calculation of the next, subleading orders.

The leading exponential factor in (32) and (34), has a clear physical interpretation: Because of the specific form of the infinite repulsion in (24), up-spin electrons are only scattered by down-spin electrons. This is reflected in the fact that the correlation length is $1 / D_{\downarrow}$. The expression $1 / D_{\downarrow}$ may be interpreted as the mean free path of the up-spin electrons. Thus the correlation length for upspin electrons is equal to their mean free path. The exponential decay of the two-particle Green functions means that, due to the strong interaction, an up-spin electron is confined by the cloud of surrounding down-spin electrons. Thus we are facing an interesting situation: Although at small distances the electrons look like free Fermions, they are confined on a macroscopic scale set by the mean free path $1 / D_{\downarrow}$. 


\section{Outline of the derivation}

The derivation of the above results on the asymptotics of the two-particle Green functions at low temperature is based on the fact that the impenetrable electron gas model is exactly solvable by Bethe ansatz. The Bethe ansatz eigenfunctions [1], 2] and the thermodynamics of the model [16] are known since long. But only recently a determinant representation for the two-particle Green functions was derived by Izergin and Pronko [17, [18]. Their derivation includes the following steps:

(i) A change of basis for the spin part of the Bethe ansatz wave function from inhomogeneous XXX to XX spin chain eigenfunctions, which is possible at infinite repulsion.

(ii) The calculation of form factors in the finite volume.

(iii) A summation of the form factors.

(iv) The thermodynamic limit.

The details of the calculation can be found in the article [18].

The asymptotic analysis of the correlation functions was performed in [4, 5]]. Starting point was the determinant representation of Izergin and Pronko (section (7), which is valid for all $x$ and $t$. The non-trivial ingredients of the determinant representation are certain auxiliary functions $b_{++}$and $B_{--}$and the Fredholm determinant $\operatorname{det}(\hat{I}+\hat{V})$ of an integral operator $\hat{V}$. A direct, yet lengthy calculation shows that $b_{++}$and $B_{--}$satisfy the separated non-linear Schrödinger equation (section 8), which is a well-known integrable partial differential equation. The logarithm of the Fredholm determinant plays the role of its tau-function (section 9). Moreover, a Riemann-Hilbert problem that fixes $b_{++}$and $B_{--}$as solutions of the separated non-linear Schrödinger equation can be derived from the determinant representation (section 10). The Riemann-Hilbert problem is the appropriate starting point for the asymptotic analysis of the correlation functions $G_{\uparrow \uparrow}^{ \pm}$(section 11).

Luckily, the differential equation and the Riemann-Hilbert problem turn out to be of the same form as in case of the impenetrable (spinless) Bose gas [19, 20]. Therefore a theorem obtained in the asymptotic analysis of the impenetrable Bose gas [21] could be applied to the impenetrable electrons as well. In contrast to the bosonic case, there is, however, an additional external integration in the determinant representation of the impenetrable electron gas. This integration can be carried out in the low temperature limit, by the method of steepest descent (section 12). 


\section{Determinant representation}

Let us recall the determinant representation for the correlation functions $G_{\uparrow \uparrow}^{ \pm}(x, t)$, which was derived in [17, 18]. We shall basically follow the account of [ [ $]$. Yet, it turns out to be useful for further calculations to rescale the variables and the correlation functions. The rescaling

$$
\begin{aligned}
& x_{r}=-\sqrt{T} x / 2, \quad t_{r}=T t / 2, \\
& g^{ \pm}=G_{\uparrow \uparrow}^{ \pm} / \sqrt{T}, \\
& \beta=\mu_{e f f} / T \quad, \quad h=B / T
\end{aligned}
$$

removes the explicit temperature dependence from all expressions. Furthermore, it will allow us to make close contact with results which were obtained for the impenetrable Bose gas [19, 21, 20]. The index ' $r$ ' in (36) stands for 'rescaled'. For the sake of simplicity we shall suppress this index in the following sections. We shall come back to physical space and time variables only in the last section, where we consider the low temperature limit.

The rescaled correlation functions $g^{+}$and $g^{-}$in the rescaled variables can be expressed as [4, 5],

$$
\begin{aligned}
& g^{+}(x, t)=\frac{-e^{2 \mathrm{i} t(\beta-h-\ln (2 \operatorname{ch}(h)))}}{2 \pi} \int_{-\pi}^{\pi} d \eta \frac{F(\gamma, \eta)}{1-\cos (\eta)} b_{++} \operatorname{det}(\hat{I}+\hat{V}), \\
& g^{-}(x, t)=\frac{e^{-2 \mathrm{i} t(\beta-h-\ln (2 \operatorname{ch}(h)))}}{4 \pi \gamma} \int_{-\pi}^{\pi} d \eta F(\gamma, \eta) B_{--} \operatorname{det}(\hat{I}+\hat{V}) .
\end{aligned}
$$

Here $\gamma$ and $F(\gamma, \eta)$ are elementary functions,

$$
\begin{gathered}
\gamma=1+e^{2 h}, \\
F(\gamma, \eta)=1+\frac{e^{i \eta}}{\gamma-e^{i \eta}}+\frac{e^{-i \eta}}{\gamma-e^{-i \eta}} .
\end{gathered}
$$

$\operatorname{det}(\hat{I}+\hat{V})$ is the Fredholm determinant of the integral operator $\hat{I}+\hat{V}$, where $\hat{I}$ is the identity operator, and $\hat{V}$ is defined by its kernel $V(\lambda, \mu)$. $\lambda$ and $\mu$ are complex variables, and the path of integration is the real axis. In order to define $V(\lambda, \mu)$ we have to introduce certain auxiliary functions. Let us define

$$
\begin{aligned}
\tau(\lambda) & =\mathrm{i}\left(\lambda^{2} t+\lambda x\right) \\
\vartheta(\lambda) & =\frac{1}{1+e^{\lambda^{2}-\beta}}, \\
E(\lambda) & =\text { p.v. } \int_{-\infty}^{\infty} d \mu \frac{e^{-2 \tau(\mu)}}{\pi(\mu-\lambda)},
\end{aligned}
$$




$$
\begin{aligned}
& e_{-}(\lambda)=\sqrt{\frac{\vartheta(\lambda)}{\pi}} e^{\tau(\lambda)}, \\
& e_{+}(\lambda)=\frac{1}{2} \sqrt{\frac{\vartheta(\lambda)}{\pi}} e^{-\tau(\lambda)}\left\{(1-\cos (\eta)) e^{2 \tau(\lambda)} E(\lambda)+\sin (\eta)\right\} .
\end{aligned}
$$

Note that $\vartheta(\lambda)$ is the Fermi weight. $V(\lambda, \mu)$ can be expressed in terms of $e_{+}$ and $e_{-}$,

$$
V(\lambda, \mu)=\frac{e_{+}(\lambda) e_{-}(\mu)-e_{+}(\mu) e_{-}(\lambda)}{\lambda-\mu} .
$$

Denote the resolvent of $\hat{V}$ by $\hat{R}$,

$$
(\hat{I}+\hat{V})(\hat{I}-\hat{R})=(\hat{I}-\hat{R})(\hat{I}+\hat{V})=\hat{I} .
$$

Then $\hat{R}$ is an integral operator with symmetric kernel [19],

$$
R(\lambda, \mu)=\frac{f_{+}(\lambda) f_{-}(\mu)-f_{+}(\mu) f_{-}(\lambda)}{\lambda-\mu},
$$

which is of the same form as $V(\lambda, \mu)$. The functions $f_{ \pm}$are obtained as the solutions of the integral equations

$$
f_{ \pm}(\lambda)+\int_{-\infty}^{\infty} d \mu V(\lambda, \mu) f_{ \pm}(\mu)=e_{ \pm}(\lambda) .
$$

We may now define the potentials

$$
B_{a b}=\int_{-\infty}^{\infty} d \lambda e_{a}(\lambda) f_{b}(\lambda) \quad, \quad C_{a b}=\int_{-\infty}^{\infty} d \lambda \lambda e_{a}(\lambda) f_{b}(\lambda)
$$

for $a, b= \pm$. $B_{--}$enters the definition of $g^{-}(x, t)$, equation (40). $b_{++}$in (39) is defined as

$$
b_{++}=B_{++}-G(x, t),
$$

where

$$
G(x, t)=\frac{(1-\cos (\eta)) e^{-\mathrm{i} \pi / 4}}{2 \sqrt{2 \pi t}} e^{\mathrm{ix} x^{2} / 2 t} .
$$

The remaining potentials $B_{a b}$ and $C_{a b}$ will be needed later.

It is instructive to compare the determinant representation (39) for the correlation function $g^{+}(x, t)$ with the corresponding expression for impenetrable Bosons (cf e.g. page 345 of [20]). The main formal differences are the occurrence of the $\eta$-integral in (39) and the occurrence of $\eta$ in the definition of $e_{+}$. As can be seen from the derivation of (39) in [18], the $\eta$-integration is related to the spin degrees of freedom. For $\eta= \pm \pi$ the expression $-\frac{1}{2} e^{2 \mathrm{i} \beta t} b_{++} \operatorname{det}(\hat{I}+\hat{V})$ agrees with the field-field correlator for impenetrable Bosons (recall, however, the different physical meaning of $\beta$ ). 


\section{Differential equations}

As in case of impenetrable Bosons [19, 20] it is possible to derive a set of integrable nonlinear partial differential equations for the potentials $b_{++}$and $B_{--}$and to express the logarithmic derivatives of the Fredholm determinant $\operatorname{det}(\hat{I}+\hat{V})$ in terms of the potentials $B_{a b}$ and $C_{a b}$.

The functions $f_{ \pm}$satisfy linear differential equations with respect to the variables $x, t$, and $\beta$,

$$
\hat{L}\left(\begin{array}{l}
f_{+} \\
f_{-}
\end{array}\right)=\hat{M}\left(\begin{array}{l}
f_{+} \\
f_{-}
\end{array}\right)=\hat{N}\left(\begin{array}{l}
f_{+} \\
f_{-}
\end{array}\right)=0,
$$

The Lax operators $\hat{L}, \hat{M}$ and $\hat{N}$ are given as

$$
\begin{aligned}
\hat{L} & =\partial_{x}+\mathrm{i} \lambda \sigma^{z}-2 \mathrm{i} Q, \\
\hat{M} & =\partial_{t}+\mathrm{i} \lambda^{2} \sigma^{z}-2 \mathrm{i} \lambda Q+\partial_{x} U, \\
\hat{N} & =2 \lambda \partial_{\beta}+\partial_{\lambda}+2 \mathrm{i} t \lambda \sigma^{z}+\mathrm{i} x \sigma^{z}-4 \mathrm{i} t Q-2 \partial_{\beta} U,
\end{aligned}
$$

where the matrices $Q$ and $U$ are defined according to

$$
Q=\left(\begin{array}{cc}
0 & b_{++} \\
B_{--} & 0
\end{array}\right) \quad, \quad U=\left(\begin{array}{cc}
-B_{+-} & b_{++} \\
-B_{--} & B_{+-}
\end{array}\right) .
$$

Mutual compatibility of the linear differential equations (55) leads to a set of nonlinear partial differential equations for the potentials $b_{++}$and $B_{--}$[5]. In particular, the space and time evolution is driven by the separated nonlinear Schrödinger equation,

$$
\begin{aligned}
& \mathrm{i} \partial_{t} b_{++}=-\frac{1}{2} \partial_{x}^{2} b_{++}-4 b_{++}^{2} B_{--} \\
& \mathrm{i} \partial_{t} B_{--}=\frac{1}{2} \partial_{x}^{2} B_{--}+4 B_{--}^{2} b_{++} .
\end{aligned}
$$

\section{Connection between Fredholm determinant and potentials}

To describe the correlation functions (39) and (40) one has to relate the Fredholm determinant $\operatorname{det}(\hat{I}+\hat{V})$ and the potentials $B_{a b}$ and $C_{a b}$. Let us use the abbreviation

$\sigma(x, t, \beta)=\ln \operatorname{det}(\hat{I}+\hat{V})$. The logarithmic derivatives of the Fredholm determinant with respect to $x, t$ and $\beta$ are

$$
\begin{aligned}
\partial_{x} \sigma= & -2 \mathrm{i} B_{+-} \\
\partial_{t} \sigma= & -2 \mathrm{i}\left(C_{+-}+C_{-+}+G(x, t) B_{--}\right) \\
\partial_{\beta} \sigma= & -2 \mathrm{i} t \partial_{\beta}\left(C_{+-}+C_{-+}+G(x, t) B_{--}\right)-2 \mathrm{i} x \partial_{\beta} B_{+-}-2\left(\partial_{\beta} B_{+-}\right)^{2} \\
& -2 \mathrm{i} t\left(B_{--} \partial_{\beta} b_{++}-b_{++} \partial_{\beta} B_{--}\right)+2\left(\partial_{\beta} b_{++}\right)\left(\partial_{\beta} B_{--}\right) .
\end{aligned}
$$


For the calculation of the asymptotics of the Fredholm determinant we further need the second derivatives of $\sigma$ with respect to space and time,

$$
\begin{aligned}
\partial_{x}^{2} \sigma & =4 B_{--} b_{++}, \\
\partial_{x} \partial_{t} \sigma & =2 \mathrm{i}\left(B_{--} \partial_{x} b_{++}-b_{++} \partial_{x} B_{--}\right), \\
\partial_{t}^{2} \sigma & =2 \mathrm{i}\left(B_{--} \partial_{t} b_{++}-b_{++} \partial_{t} B_{--}\right)+8 B_{--}^{2} b_{++}^{2}+2\left(\partial_{x} B_{--}\right)\left(\partial_{x} b_{++}\right) .
\end{aligned}
$$

Note that

$$
\lim _{\beta \rightarrow-\infty} \sigma=0 .
$$

This follows from $\lim _{\beta \rightarrow-\infty} \vartheta(\lambda)=0$ and is important for fixing the integration constant in the calculation of the asymptotics of the determinant.

\section{The Riemann-Hilbert problem}

From now on we will restrict ourselves to the case of negative effective chemical potential, $\beta<0$. Recall that this is the condition for the system to be in the gas phase. For negative $\beta$ the logarithmic derivatives $\partial_{x} \sigma$ and $\partial_{t} \sigma$ of the Fredholm determinant and the potentials $b_{++}$and $B_{--}$are determined by the following matrix Riemann-Hilbert problem, which was derived from the determinant representation (see section 7) in [П].

(i) $\phi: \mathbb{C} \rightarrow \operatorname{End}\left(\mathbb{C}^{2}\right)$ is analytic in $\mathbb{C} \backslash \mathbb{R}$.

(ii) $\lim _{\lambda \rightarrow \infty} \phi(\lambda)=I_{2}$.

(iii) $\phi$ has a discontinuity across the real axis described by the condition

$$
\phi_{-}(\lambda)=\phi_{+}(\lambda)\left(\begin{array}{cc}
1 & p(\lambda) e^{-2 \tau(\lambda)} \\
q(\lambda) e^{2 \tau(\lambda)} & 1+p(\lambda) q(\lambda)
\end{array}\right)
$$

for all $\lambda \in \mathbb{R}$.

Here $I_{2}$ denotes the $2 \times 2$ unit matrix. The functions $p(\lambda)$ and $q(\lambda)$ are defined as

$$
\begin{aligned}
& p(\lambda)=\mathrm{i}(\cos (\eta)-1)(1-\vartheta(\lambda)) \alpha_{+}(\lambda) \alpha_{-}(\lambda), \\
& q(\lambda)=-\frac{2 \mathrm{i} \vartheta(\lambda)}{\alpha_{+}(\lambda) \alpha_{-}(\lambda)},
\end{aligned}
$$

where

$$
\alpha(\lambda)=\exp \left\{-\frac{1}{2 \pi \mathrm{i}} \int_{-\infty}^{\infty} \frac{d \mu}{\mu-\lambda} \ln \left(1+\vartheta(\mu)\left(e^{-\mathrm{i \eta}}-1\right)\right)\right\} .
$$


The functions $\partial_{x} \sigma, \partial_{t} \sigma, b_{++}$and $B_{--}$can be expressed through the coefficients in the asymptotic expansions of $\phi(\lambda)$ and $\ln (\alpha(\lambda))$ for large spectral parameter $\lambda$. Let

$$
\phi(\lambda)=I_{2}+\frac{\phi^{(1)}}{\lambda}+\frac{\phi^{(2)}}{\lambda^{2}}+O\left(\frac{1}{\lambda^{3}}\right)
$$

and

$$
\ln (\alpha(\lambda))=\frac{\alpha_{1}}{\lambda}+\frac{\alpha_{2}}{\lambda^{2}}+O\left(\frac{1}{\lambda^{3}}\right)
$$

Then

$$
\begin{array}{ccc}
\partial_{x} \sigma=2 \mathrm{i} \alpha_{1}+\mathrm{i} \operatorname{tr}\left\{\phi^{(1)} \sigma^{z}\right\} & , \quad \partial_{t} \sigma=4 \mathrm{i} \alpha_{2}+2 \mathrm{i} \operatorname{tr}\left\{\phi^{(2)} \sigma^{z}\right\}, \\
b_{++}=\phi_{12}^{(1)} & , \quad B_{--}=-\phi_{21}^{(1)} .
\end{array}
$$

The Riemann-Hilbert problem is the appropriate starting point for the asymptotic analysis of the potentials $b_{++}$and $B_{--}$which determine the asymptotics of the two-particle Green functions $G_{\uparrow}^{ \pm}$. For impenetrable Bosons a similar analysis was carried out in [21]. Fortunately, the result of [21] depends only on some general properties of the functions $p(\lambda)$ and $q(\lambda)$ entering the conjugation matrix in (69), and also applies in the present case. Alternatively, the non-linear steepest descent method of Deift and Zhou [22] could be applied.

\section{Asymptotics of the correlation functions}

The direct asymptotic analysis of the Riemann-Hilbert problem yields the leading order asymptotics $\left(x, t \rightarrow \infty\right.$ for fixed ratio $\left.\lambda_{0}=-2 x / t\right)$ of the functions $\partial_{x} \sigma, \partial_{t} \sigma$, $b_{++}$and $B_{--}$[5, 21]. It turns out, in particular, that $b_{++}$and $B_{--}$are a decaying solution of the separated nonlinear Schrödinger equation (60), (61). Now the form of the complete asymptotic decomposition of the decaying solutions of the separated nonlinear Schrödinger equation is known [23, 24].

$$
\begin{aligned}
& b_{++}=t^{-\frac{1}{2}}\left(u_{0}+\sum_{n=1}^{\infty} \sum_{k=0}^{2 n} \frac{\ln ^{k} 4 t}{t^{n}} u_{n k}\right) \exp \left\{\frac{\mathrm{i} x^{2}}{2 t}-\mathrm{i} v \ln 4 t\right\}, \\
& B_{--}=t^{-\frac{1}{2}}\left(v_{0}+\sum_{n=1}^{\infty} \sum_{k=0}^{2 n} \frac{\ln ^{k} 4 t}{t^{n}} v_{n k}\right) \exp \left\{-\frac{\mathrm{i} x^{2}}{2 t}+\mathrm{i} v \ln 4 t\right\},
\end{aligned}
$$

where $u_{0}, v_{0}, u_{n k}, v_{n k}$ and $v$ are functions of $\lambda_{0}=-x / 2 t$ and of $\beta$ and $\eta$. Inserting the asymptotic expansions for $B_{--}$and $b_{++}$into the differential equations (60), 
(61) we obtain expressions for $u_{n k}, v_{n k}$ and $v$ in terms of $u_{0}$ and $v_{0}$, i.e. the two unknown functions $u_{0}$ and $v_{0}$ determine the whole asymptotic expansion (77), (78). But $u_{0}$ and $v_{0}$ are obtained from the asymptotic analysis of the Riemann-Hilbert problem (for the explicit expressions see [5]). Hence we know, in principle, the complete asymptotic decomposition of the potentials $b_{++}$and $B_{--}$.

In order to obtain the asymptotics of the two-particle Green functions we still need the asymptotics of the Fredholm determinant. The Fredholm determinant is related to $b_{++}$and $B_{--}$through equations (65)-(67) and (62)-(64). We may integrate (65)-(67) to obtain the asymptotic expansions of $\partial_{x} \sigma$ and $\partial_{t} \sigma$. The integration constant is a function of $\beta$. It is fixed by the leading asymptotics, which, using (75), can be obtained from the direct asymptotic analysis of the RiemannHilbert problem. Then, integrating (62)-(64) yields $\sigma$ up to a numerical constant, which follows from the asymptotic condition (68). The calculation is the same as for the impenetrable Bose gas and can be found on pages 455-457 of [20].

Finally, we obtain the following expressions for the leading asymptotics of the correlation function,

$$
\begin{aligned}
g^{+}(x, t)= & e^{\mathrm{i} x^{2} / 2 t+2 \mathrm{it} \beta} e^{-2 \mathrm{it}(h+\ln (2 \mathrm{ch}(h)))} \int_{-\pi}^{\pi} d \eta \frac{F(\gamma, \eta)}{1-\cos (\eta)} . \\
& \cdot C^{+}\left(\lambda_{0}, \beta, \eta\right)(4 t)^{\frac{1}{2}(v-\mathrm{i})^{2}} \exp \left\{\frac{1}{\pi} \int_{-\infty}^{\infty} d \lambda|x+2 \lambda t| \ln (\varphi(\lambda, \beta))\right\}, \\
g^{-}(x, t)= & e^{-\mathrm{ix} x^{2} / 2 t-2 \mathrm{it} \beta} e^{2 \mathrm{it}(h+\ln (2 \mathrm{ch}(h)))} \int_{-\pi}^{\pi} d \eta \frac{F(\gamma, \eta)}{2 \gamma} . \\
& \cdot C^{-}\left(\lambda_{0}, \beta, \eta\right)(4 t)^{\frac{1}{2}(v+\mathrm{i})^{2}} \exp \left\{\frac{1}{\pi} \int_{-\infty}^{\infty} d \lambda|x+2 \lambda t| \ln (\varphi(\lambda, \beta))\right\},
\end{aligned}
$$

where

$$
\begin{aligned}
& \varphi(\lambda, \beta)=1+\vartheta(\lambda)\left(e^{-\mathrm{in} \operatorname{sign}\left(\lambda-\lambda_{0}\right)}-1\right) \\
& v=-\frac{1}{2 \pi} \ln \left(1-2(1-\cos (\eta)) \vartheta\left(\lambda_{0}\right)\left(1-\vartheta\left(\lambda_{0}\right)\right)\right) \\
& C^{+}\left(\lambda_{0}, \beta, \eta\right)=-\mid \sin (\eta / 2) \mid \frac{\sqrt{v}}{2 \pi} \exp \left\{\frac{1}{2}\left(\lambda_{0}^{2}-\beta\right)+\mathrm{i} \Psi_{0}+\frac{v^{2}}{2}\right. \\
&-\int_{-\infty}^{\beta} d \beta\left(\mathrm{iv} / 2+v \partial_{\beta} \Psi_{0}\right) \\
&\left.+\frac{1}{2 \pi^{2}} \int_{-\infty}^{\beta} d \beta\left(\partial_{\beta} \int_{-\infty}^{\infty} d \lambda \operatorname{sign}\left(\lambda-\lambda_{0}\right) \ln (\varphi(\lambda, \beta))\right)^{2}\right\} \\
& C^{-}\left(\lambda_{0}, \beta, \eta\right)=C^{+}\left(\lambda_{0}, \beta, \eta\right) \exp \left(-\left(\lambda_{0}^{2}-\beta\right)-2 \mathrm{i} \Psi_{0}\right) / \sin ^{2}(\eta / 2)
\end{aligned}
$$


$\lambda_{0}=-x / 2 t$ is the stationary point of the phase $\tau(\lambda)$ (i.e. $\tau^{\prime}\left(\lambda_{0}\right)=0$ ), and the functions $\Psi_{0}$ and $\Psi_{1}$ are defined as

$$
\begin{aligned}
& \Psi_{0}=-\frac{3 \pi}{4}+\arg \Gamma(\mathrm{iv})+\Psi_{1} \\
& \Psi_{1}=-\frac{1}{\pi} \int_{-\infty}^{\infty} d \lambda \operatorname{sign}\left(\lambda-\lambda_{0}\right) \ln \left|\lambda-\lambda_{0}\right| \partial_{\lambda} \ln (\varphi(\lambda, \beta)) .
\end{aligned}
$$

Equations (79) and (80) are valid for large $t$ and fixed finite ratio $\lambda_{0}=-x / 2 t$. Correlations in the pure space direction $t=0$ were discussed in [25]. We would like to emphasize that (79) and (80) still hold for arbitrary temperatures. The low temperature limit will be discussed in the next section. Note that there is no pole of the integrand at $\eta=0$, since $\sqrt{v} \sim|\eta|$ for small $\eta$ and thus $C^{+}\left(\lambda_{0}, \beta, \eta\right) \sim \eta^{2}$.

\section{Asymptotics in the low temperature limit}

For the following steepest descent calculation we transform the $\eta$-integrals in (79), (80) into complex contour integrals over the the unit circle, setting $z=e^{i \eta}$. Since we would like to consider low temperatures, we have to restore the explicit temperature dependence by scaling back to the physical space and time variables $x$ and $t$ and to the physical correlation functions $G_{\uparrow \uparrow}^{ \pm}$. Recall that in the previous sections we have suppressed an index ' $r$ ' referring to 'rescaled'. Let us restore this index in order to define $k_{0}=\lambda_{0} \sqrt{T}=x / 2 t, \vartheta(k)=\vartheta_{r}(k / \sqrt{T}), \varphi(k, \beta)=\varphi_{r}(k / \sqrt{T}, \beta)$, $C^{ \pm}\left(k_{0}, \beta, z\right)=C_{r}^{ \pm}\left(\lambda_{0}, \beta, \eta\right), F(\gamma, z)=F_{r}(\gamma, \eta)$. Then

$$
\begin{aligned}
& G_{\uparrow \uparrow}^{+}(x, t)=2 \mathrm{i} \sqrt{T} e^{\mathrm{i} x^{2} / 4 t+\mathrm{i} t(\mu-B)} \oint d z \frac{F(\gamma, z)}{(z-1)^{2}} C^{+}\left(k_{0}, \beta, z\right)(2 T t)^{\frac{1}{2}(v(z)-\mathrm{i})^{2}} e^{t S(z)}, \\
& G_{\uparrow \uparrow}^{-}(x, t)=-\mathrm{i} \sqrt{T} e^{-\mathrm{i} x^{2} / 4 t-\mathrm{i} t(\mu-B)} \oint d z \frac{F(\gamma, z)}{2 \gamma z} C^{-}\left(k_{0}, \beta, z\right)(2 T t)^{\frac{1}{2}(v(z)+\mathrm{i})^{2}} e^{t S(z)},
\end{aligned}
$$

where

$$
S(z)=\frac{1}{\pi} \int_{-\infty}^{\infty} d k\left|k-k_{0}\right| \ln (\varphi(k, \beta))
$$

We would like to calculate the contour integrals $(87),(88)$ by the method of steepest descent. For this purpose we have to consider the analytic properties of the integrands. Let us assume that $k_{0} \geq 0$, and let us cut the complex plane along the real axis from $-\infty$ to $-e^{-\beta}$ and from $-e^{\beta-k_{0}^{2} / T}$ to 0 . The integrands in 87) and (88) can be analytically continued as functions of $z$ into the cut plane with the 
only exception of the two simple poles of $F(\gamma, z)$ at $z=\gamma^{ \pm 1}$. We may therefore deform the contour of integration as long as we never cross the cuts and take into account the pole contributions, if we cross $z=\gamma$ or $z=\gamma^{-1}$.

The saddle point equation $\partial S / \partial z=0$ can be represented in the form

$$
\int_{0}^{\infty} \frac{d k k}{1+z^{-1} e^{-\beta} e^{\left(k-k_{0}\right)^{2} / T}}=\int_{0}^{\infty} \frac{d k k}{1+z e^{-\beta} e^{\left(k+k_{0}\right)^{2} / T}} .
$$

This equation was discussed in the appendix of [4]. In [ [7] it was shown that (90) has exactly one real positive solution which is located in the interval $[0,1]$. It was argued that this solution gives the leading saddle point contribution to (87) and (88). At small temperatures (90) can be solved explicitly. There are two solutions $z_{ \pm}= \pm z_{c}$, where

$$
z_{c}=\frac{T^{3 / 4}}{2 \pi^{1 / 4} k_{0}^{3 / 2}} e^{-k_{0}^{2} / 2 T} .
$$

In the derivation of (91) we assumed that $k_{0} \neq 0$. The case $k_{0}=0$ has to be treated separately (see below).

The phase $t S(z)$ has the low temperature approximation

$$
t S(z)=-2 k_{0} D t\left\{\left(1-\frac{1}{z}\right) z_{c}^{2}+1-z\right\} .
$$

Here $D=\partial P / \partial \mu$ is the density of the electron gas. The low temperature expansion (92) is valid in an annulus $e^{\beta-k_{0}^{2} / T} \ll|z| \ll e^{-\beta}$, which lies in our cut plane. The unit circle and the circle $|z|=z_{c}$ are inside this annulus. We may thus first apply (92) and then deform the contour of integration from the unit circle to the small circle $|z|=z_{c}$. Let us parameterize the small circle as $z=z_{c} e^{\mathrm{i} \alpha}, \alpha \in[-\pi, \pi]$. Then $S(z(\alpha))=-2 k_{0} D\left(\left(z_{c}-1\right)^{2}+2 z_{c}(1-\cos (\alpha))\right)$, which implies that the small circle is a steepest descent contour and that on this contour $S\left(z_{-}\right) \leq S(z) \leq S\left(z_{+}\right)$. The maximum of $S(z)$ on the steepest descent contour at $z=z_{+}$is unique and therefore provides the leading saddle point contribution to (87), (88) as $t \rightarrow \infty$. The saddle point approximation becomes good when $t S(z(\alpha))=-2 k_{0} D t\left(\left(z_{c}-1\right)^{2}+z_{c} \alpha^{2}+\right.$ $O\left(\alpha^{4}\right)$ ) becomes sharply peaked around $\alpha=0$. Hence, the relevant parameter for the calculation of the asymptotics of $G_{\uparrow \uparrow}^{ \pm}$is $2 k_{0} D t=x D$ rather than $t . x D$ has to be large compared to $z_{c}^{-1}$. The parameter $x D$ has a simple interpretation. It is the average number of particles in the interval $[0, x]$. Let us consider two different limiting cases.

(i) $x D \rightarrow 0$, the number of electrons in the interval $[0, x]$ vanishes. In this regime the interaction of the electrons is negligible. An electron propagates freely 
from 0 to $x$. $G_{\uparrow \uparrow}^{ \pm}$cannot be calculated by the method of steepest descent. We have to use the integral representation (79), (80) instead. Since $t S(z)$ and $v(z)$ tend to zero on the contour of integration, the integrals in (79) and (80) are easily calculated. We find $G_{\uparrow \uparrow}^{ \pm}=G_{f}^{ \pm}$(see (30), (31)), which is the well known result for free Fermions.

(ii) $x D \gg z_{c}^{-1}$, the average number of electrons in the interval $[0, x]$ is large. This is the true asymptotic region, $x \rightarrow \infty$. In this region the interaction becomes important. At the same time the method of steepest descent can be used to calculate $G_{\uparrow \uparrow}^{ \pm}$. This case will be studied below.

In the process of deformation of the contour from the unit circle to the small circle of radius $z_{c}$ we may cross the pole of the function $F(\gamma, z)$ at $z=\gamma^{-1}$. Then we obtain a contribution of the pole to the asymptotics of $G_{\uparrow \uparrow}^{ \pm}$. It turns out that the pole contributes to $G_{\uparrow}^{ \pm}$, when the magnetic field is below a critical positive value, $B_{c}=$ $k_{0}^{2} / 4$. Below this value the pole contribution always dominates the contribution of the saddle point. Hence, we have to distinguish two different asymptotic regions, $B>B_{c}$ and $B<B_{c}$. On the other hand, if we consider the asymptotics for fixed magnetic field, we have to treat the cases $B>0$ and $B \leq 0$ separately. For $B>0$ we have to distinguish between a time like regime $\left(k_{0}^{2}<4 B\right)$ and a space like regime $\left(k_{0}^{2}>4 B\right)$. In these respective regimes we obtain the asymptotics (32), (34).

In the limit $B \rightarrow-\infty, \mu \rightarrow-\infty, \mu-B$ fixed there are no $\downarrow$-spin electrons left in the system, $D_{\downarrow} \rightarrow 0$. This is the free Fermion limit. In the free Fermion limit $B<B_{c}$, and the asymptotics of $G_{\uparrow \uparrow}^{+}(x, t)$ and $G_{\uparrow \uparrow}^{-}(x, t)$ are given by the equations (34), which turn into the expressions (30), (31) for free Fermions.

The pure time direction $k_{0}=0$ requires a separate calculation. For $k_{0}=0$ the saddle point equation (90) has the solutions $z= \pm 1$ for all temperatures. The unit circle is a steepest descent contour with unique maximum of $S(z)$ at $z=1$, which gives the leading asymptotic contribution to the integrals in (87) and (88). We find algebraically decaying correlations,

$$
G_{\uparrow \uparrow}^{+}(0, t)=C_{0}^{+} t^{-1} e^{\mathrm{i} t(\mu-B)} \quad, \quad G_{\uparrow \uparrow}^{-}(0, t)=C_{0}^{-} t^{-1} e^{-\mathrm{i} t(\mu-B)},
$$

where

$$
\begin{aligned}
C_{0}^{+}= & \frac{e^{-\mathrm{i} \frac{\pi}{4}}}{2 \sqrt{2 \pi T}}\left(1+2 e^{-2 B / T}\right) \\
& {\left[\left(e^{(\mu+B) / T}+e^{(\mu-B) / T}\right)\left(1+e^{(\mu+B) / T}+e^{(\mu-B) / T}\right)\right]^{-\frac{1}{2}}, } \\
C_{0}^{-}= & \frac{e^{\mathrm{i} \frac{\pi}{4}}}{2 \sqrt{2 \pi T}} \frac{1+2 e^{-2 B / T}}{1+e^{2 B / T}}\left[\frac{e^{(\mu+B) / T}+e^{(\mu-B) / T}}{1+e^{(\mu+B) / T}+e^{(\mu-B) / T}}\right]^{\frac{1}{2}} .
\end{aligned}
$$

These formulae are valid at any temperature. 


\section{Acknowledgment}

The results presented in this talk were obtained in collaboration with A. R. Its and V. E. Korepin to whom the author would like to express his deep gratitude. The author thanks W. Pesch for a critical reading of the manuscript. He was supported by H. Fehske and the DFG Schwerpunktprogramm SPP 1073 'Kollektive Quantenzustände in elektronisch eindimensionalen Übergangsmetallverbindungen'.

\section{References}

[1] C. N. Yang, Phys. Rev. Lett. 19, 1312 (1967).

[2] M. Gaudin, Phys. Lett. A 24, 55 (1967).

[3] F. Göhmann and V. E. Korepin, Phys. Lett. A 260, 516 (1999).

[4] F. Göhmann, A. G. Izergin, V. E. Korepin, and A. G. Pronko, Int. J. Mod. Phys. B 12, 2409 (1998).

[5] F. Göhmann, A. R. Its, and V. E. Korepin, Phys. Lett. A 249, 117 (1998).

[6] F. D. M. Haldane, Phys. Rev. Lett. 45, 1358 (1980).

[7] F. D. M. Haldane, J. Phys. C 14, 2585 (1981).

[8] J. Voit, Rep. Prog. Phys. 57, 977 (1994).

[9] A. A. Belavin, A. M. Polyakov, and A. B. Zamolodchikov, Nucl. Phys. B 241, 333 (1984).

[10] E. H. Lieb and F. Y. Wu, Phys. Rev. Lett. 20, 1445 (1968).

[11] H. Frahm and V. E. Korepin, Phys. Rev. B 42, 10553 (1990).

[12] H. Frahm and V. E. Korepin, Phys. Rev. B 43, 5653 (1991).

[13] T. Deguchi, F. H. L. Essler, F. Göhmann, A. Klümper, V. E. Korepin, and K. Kusakabe, Phys. Rep. 331, 197 (2000).

[14] M. Takahashi, Prog. Theor. Phys. 47, 69 (1972).

[15] M. Takahashi, Prog. Theor. Phys. 52, 103 (1974).

[16] M. Takahashi, Prog. Theor. Phys. 46, 1388 (1971).

[17] A. G. Izergin and A. G. Pronko, Phys. Lett. A 236, 445 (1997). 
[18] A. G. Izergin and A. G. Pronko, Nucl. Phys. B 520, 594 (1998).

[19] A. R. Its, A. G. Izergin, V. E. Korepin, and N. Slavnov, Int. J. Mod. Phys. B 4, 1003 (1990).

[20] V. E. Korepin, N. M. Bogoliubov, and A. G. Izergin, Quantum Inverse Scattering Method and Correlation Functions, Cambridge University Press, (1993).

[21] A. R. Its, A. G. Izergin, V. E. Korepin, and G. G. Varzugin, Physica D 54, 351 (1992).

[22] P. A. Deift and X. Zhou, Ann. of Math. 137, 295 (1993).

[23] H. Segur and M. J. Ablowitz, J. Math. Phys. 17, 710 (1976).

[24] M. J. Ablowitz and H. Segur, Solitons and the Inverse Scattering Transform, SIAM, Philadelphia, (1981).

[25] A. Berkovich, J. Phys. A 24, 1543 (1991). 